\title{
A UNIFORM SET COVERING LEMMA ${ }^{1}$
}

\author{
DAVID W. MATULA
}

ABSTRACT. The bounded set system $H=(V, \mathcal{F})$ is composed of a nonvoid set $V$ and a set, $\mathcal{F}$, of nonvoid subsets of $V$, a finite number of which cover $V . C \subset V$ is a critical subset of $H$ if every proper subset of $C$ requires fewer members of $\mathcal{F}$ to cover it than are needed to cover $C$. For $|\mathcal{F}|$ finite, it is shown that every $A \subset V$ contains a critical $C \subset A$ requiring the same number of members of $\mathcal{F}$ in a minimum cover. For $v \in V, l(v)$ is the largest number of members of $\mathcal{F}$ in any minimum cover of any critical set containing $v$. For $|\mathfrak{F}|$ finite, it is shown that there exists a covering $A_{1}, A_{2}, \cdots, A_{k}, A_{i} \in \mathcal{F}$ for $1 \leq i \leq k$, such that $v \epsilon$ $\bigcup_{i=1}^{l(v)} A_{i}$ for all $v \in V$. An application to graph coloring is described.

I. Coverings and critical sets. The set system (or hypergraph [1]) $H=$ $(V, \mathfrak{F})$ is composed of a nonvoid set $V$ and a set, $\mathfrak{F}$, of nonvoid subsets of $V$ that cover $V . H=(V, \mathfrak{F})$ is a bounded set system if a finite number of members of $\mathcal{F}$ cover $V$, and $H$ is a finite set system if $|V|$ is finite. A set system is necessarily bounded if either $|V|$ or $|\mathfrak{F}|$ is finite. For the bounded set system $H=(V, \mathfrak{F})$, the covering number, $\chi_{H}(A)$, of $A \subset V$ is the minimum number of members of $\mathfrak{F}$ whose union contains $A$, and $A \subset V$ is k-partite in $H$ if $\chi_{H}(A) \leq k$. The subset $C \subset V$ is critical in the bounded set system $H$ if $\chi_{H}(A) \leq \chi_{H}(C)-1$ for all $A \subset C, A \not C$. Note that $\{v\}$ is critical for every $v \in V$.

For a finite set system $H=(V, \mathcal{F})$ it is clear that if $\chi_{H}(A)=n$, then there exists a critical $C \subset A$ such that $\chi_{H}(C)=\chi_{H}(A)=n$. This result is still obtained if only $\mathcal{F}$ is assumed finite.

Lemma 1. For the set system $H=(V, \mathcal{F})$ where $|\mathfrak{F}|=q$, any $A \subset V$ contains a critical $C \subset A$ with $\chi_{H}(C)=\chi_{H}(A)$ where

$$
|C| \leq\left(\begin{array}{c}
q \\
\chi_{H}(A)-1
\end{array}\right) \text {. }
$$

Received by the editors June 1, 1973.

AMS (MOS) subject classifications (1970). Primary 05A05; Secondary 05C15.

Key words and phrases. Set coverings, critical sets, minimum covers, uniform cover, hypergraph, graph covering, Grundy function.

1 Work was supported in part by Army Research Office (Durham) grant DAHC04 $74 \mathrm{C} 0198$ at The University of Texas at Austin. 
Proof. Let $H=(V, \mathcal{F})$ with $|\mathfrak{F}|=q$. Then for $1 \leq k \leq q$, there are at most $\left(\begin{array}{c}q \\ k\end{array}\right)$ maximal $k$-partite sets in $H$, so every $k$-partite set of $H$ is contained in a maximal $k$-partite set of $H$. For $A \subset V$ with $\chi(A)=1,\{v\}$ is critical for any $v \in A$, thus satisfying the lemma in this case. Let $A_{0} \subset V$ be chosen with $\chi_{H}\left(A_{0}\right)=k \geq 2$. Choose $B_{1}$ to be one of the maximal $(k-1)$-partite sets of $H$ such that $A_{0} \cap B_{1}$ is maximal in $A_{0}$ with $\chi\left(A_{0} \cap B_{1}\right)=k-1$. Choose $a_{1} \in A_{0}-B_{1}$, and let $A_{1}=A_{0} \cap B_{1} \cup\left\{a_{1}\right\}$, so then $\chi_{H}\left(A_{1}\right)=k$. If $\chi_{H}\left(\left\{a_{1}, a_{2}, \cdots, a_{i}\right\}\right) \leq k-1$, for $i \geq 1$, choose $B_{i+1}$ to be one of the maximal $(k-1)$-partite sets of $H$ with $\left\{a_{1}, a_{2}, \cdots, a_{i}\right\} \subset B_{i+1}$, where also $A_{i} \cap B_{i+1}$ is maximal in $A_{i}$ with $\chi_{H}\left(A_{i} \cap B_{i+1}\right)=k-1$. Choose $a_{i+1} \in A_{i}-B_{i+1}$, and let $A_{i+1}=A_{i} \cap B_{i+1} \cup\left\{a_{i+1}\right\}$, so then $\chi_{H}\left(A_{i+1}\right)=k$. Thus when $\chi_{H}\left(\left\{a_{1}, a_{2}, \cdots, a_{i}\right\}\right) \leq k-1, A_{0} \supset A_{1} \supset A_{2} \supset \cdots \supset A_{i} \supset A_{i+1} \supset\left\{a_{1}, a_{2}, \cdots\right.$, $\left.a_{i}, a_{i+1}\right\}$ and $\chi_{H}\left(A_{j}\right)=k$ for $0 \leq j \leq i+1$. Furthermore, the $B_{j}$ so chosen satisfy $\left\{a_{1}, a_{2}, \cdots, a_{j-1}\right\} \subset B_{j}, a_{j} \notin B_{j}$, for $1 \leq j \leq i+1$, so the $B_{j}$ are distinct maximal $(k-1)$-partite sets of $H$. Eventually for some index $n \leq\left(\begin{array}{c}q \\ k-1\end{array}\right)-1$, $\chi_{H}\left(\left\{a_{1}, a_{2}, \cdots, a_{n+1}\right\}\right)=k$. Let $C=\left\{a_{1}, a_{2}, \cdots, a_{n+1}\right\}$. Then $\chi_{H}^{-}(C)=k$ and $\chi_{H}\left(C-\left\{a_{n+1}\right\}\right)=k-1$. For $1 \leq j \leq n,\left\{a_{1}, a_{2}, \cdots, a_{j-1}\right\} \subset B_{j}$. Also $\left\{a_{j+1}, a_{j+2}, \cdots, a_{n+1}\right\} \subset A_{n+1} \subset A_{j} \subset A_{j-1}$, and since $A_{j}=A_{j-1} \cap B_{j} \cup\left\{a_{j}\right\}$, it follows that $\left\{a_{j+1}, a_{j+2}, \cdots, a_{n+1}\right\} \subset B_{j}$. Hence $C-\left\{a_{j}\right\} \subset B_{j}$, and $\chi_{H}\left(C-\left\{a_{j}\right\}\right) \leq \chi_{H}\left(B_{j}\right)=k-1$ for $1 \leq j \leq n$. So $C=\left\{a_{1}, \cdots, a_{n+1}\right\}$ is critical with $\chi_{H}(C)=\chi_{H}(A)$, and $|C|=n+1 \leq\left(\begin{array}{c}q \\ k-1\end{array}\right)$.

Coroilary 1.1. Let $H=(V, \mathcal{F})$ be a set system with $|\mathfrak{F}|=q$. Then every critical $C \subset V$ has at most

$$
\left(\begin{array}{c}
q \\
\chi_{H}(C)-1
\end{array}\right)
$$

members. Furthermore, for any $1 \leq k \leq q$, there exists an $H=(V, \mathcal{F})$ with $|\mathcal{F}|=q$ and $a$ critical $C \subset V$ with $\bar{\chi}_{H}(C)=k$ and

$$
|C|=\left(\begin{array}{c}
q \\
\chi_{H}(C)-1
\end{array}\right) \text {. }
$$

Proof. Let $H=(V, \mathcal{F})$ with $|\mathfrak{F}|=q$. For any critical $C^{\prime} \subset V$, by Lemma 1 there exists $C \subset C^{\prime}, C$ critical, $\chi_{H}(C)=\chi_{H}\left(C^{\prime}\right)$ and

$$
|C| \leq\left(\begin{array}{c}
q \\
\chi_{H}(C)-1
\end{array}\right) \text {. }
$$


Since $C^{\prime}$ is critical with $\chi_{H}(C)=\chi_{H}\left(C^{\prime}\right), C=C^{\prime}$, verifying the bound of the lemma. To show that this result is best possible, let $k$ and $q$ be given with $1 \leq k \leq q$. Let $H=(V, \mathcal{F})$ be constructed so that $|V|=\left(\begin{array}{c}q \\ k-1\end{array}\right)$, where $\mathcal{F}=$ $\left\{s_{1}, s_{2}, \cdots, s_{q}\right\}$, and each member of $V$ is contained in a unique choice of $q-k+1$ of the subsets $\left\{S_{i}\right\}, i=1, \cdots, q$. Thus any union of $k-1$ of the $q$ members of $\mathfrak{F}$ will contain all but one member of $V$, and $V-\{v\}$ will be covered by a unique set of $k-1$ of the $q$ members of $\mathfrak{F}$ for any $v \in V$. Hence $\chi_{H}(V)=k$ and $\chi_{H}(V-\{v\})=k-1$ for any $v \in V$, so $V$ is critical in $H$ with

$$
|V|=\left(\begin{array}{c}
q \\
\chi_{H}(V)-1
\end{array}\right)
$$

showing the bound is best possible.

It should be noted that the result of Lemma 1 is not obtained for all bounded set systems. For example, let $H^{\prime}=\left(V^{\prime}, \mathcal{F}^{\prime}\right)$, where

$$
V^{\prime}=\{0,1,2, \cdots\}, \quad \mathcal{F}^{\prime}=\left\{E, D, I_{1}, I_{2}, I_{3}, \cdots\right\} \text {, }
$$

where $E=\{0,2,4,6, \cdots\}, D=\{1,3,5, \cdots\}$, and $I_{j}=\{0,1,2, \cdots, j\}$ for $j \geq 1$. Any nonempty set of nonnegative integers which are all even, all odd, or finite in number will have a covering number of unity in $H^{\prime}$, and all other nonempty sets of nonnegative integers will have a covering number of two in $H^{\prime}$. Any $\{i\}, i \geq 0$, is critical in $H^{\prime}$ with covering number unity, but there are no critical sets with covering number two in $H^{\prime}$.

II. The covering level and uniform set coverings. For any bounded set system $H=(V, \mathcal{F})$, each element $v \in V$ has a covering level, $l(v)$, given by

$$
l(v) \equiv \max _{C \subset V}\left\{\chi_{H}(C) \mid v \in C, C \text { is critical }\right\},
$$

where then $1 \leq l(v) \leq \chi_{H}(V)$ for all $v \in V$.

The following lemma shows that in any bounded set system where $|\mathcal{F}|$ is finite, the covering level of $v$ is equivalent to the largest covering number of those sets in which $v$ is an "essential member."

Lemma 2. Let the set system $H=(V, \mathcal{F})$ where $|\mathcal{F}|$ is finite. For any $v \in V$,

$$
l(v)=\max _{A \subset V}\left\{\chi_{H}(A) \mid v \in A, \chi_{H}(A-\{v\}) \leq \chi_{H}(A)-1\right\}
$$

Proof. Let the set system $H=(V, \mathcal{F})$ where $|\mathcal{F}|$ is finite, so $H$ is bounded. For every critical $C \subset V$ with $v \in C$, we have $\chi_{H}(C-\{v\}) \leq \chi_{H}(C)-1$, so $l(v) \leq \max _{A \subset V}\left\{\chi_{H}(A) \mid v \in A, \chi_{H}(A-\{v\}) \leq \chi_{H}(A)-1\right\}$. Let $A^{*} \in V$ be a set 
maximizing the right-hand side of (3). If $A^{*}$ is not critical, from Lemma 1 there exists a critical $C \subset A^{*}$ with $\chi_{H}(C)=\chi_{H}\left(A^{*}\right)$. If $v \notin C$, then $C \subset A^{*}$ $\{v\}$ would imply $\chi_{H}(C) \leq \chi_{H}\left(A^{*}-\{v\}\right) \leq \chi_{H}\left(A^{*}\right)-1$, a contradiction. Thus $v$ is contained in the critical set $C$, so $l(v) \geq \chi_{H}(C)=\chi_{H}\left(A^{*}\right)=$ $\max _{A} \subset V$ $\left\{\chi_{H}(A) \mid v \in A, \chi_{H}(A-\{v\}) \leq \chi_{H}(A)-1\right\}$, proving the lemma.

Corollary 2.1. Let the set system $H=(V, \mathcal{F})$ where $|\mathfrak{F}|$ is finite. Let $v \in V$ have $l(v)=k$, and let $B_{k} \subset V$ be any maximal $k$-partite set of $H$. Then $v \in B_{k}$

Proof. For $v$ and $B_{k}$ as specified, assume $v \notin B_{k}$. Then $\chi_{H}\left(B_{k} \cup\{v\}\right)=$ $k+1$, since $B_{k}$ is maximal with $\chi_{H}\left(B_{k}\right) \leq k$. Thus, from Lemma $2, l(v) \geq$ $k+1$, a contradiction. So $v \in B_{k}$.

From Corollary 2.1 it may be concluded that when $|\mathcal{F}|$ is finite, $l(v)=1$ if and only if $v$ is in every maximal member of $\mathcal{F}$.

For the bounded set system $H=(V, \mathcal{F})$, there must exist some covering $A_{1}$, $A_{2}, \cdots, A_{k}$ of $V$ where $A_{i} \in \mathcal{F}$ for $1 \leq i \leq k$, and $k=\chi_{H}(V)$. When $|\mathcal{F}|$ is finite, the $A_{i}$ may each be assumed to be maximal members of $\mathcal{F}$, but this does not guarantee that $B=A_{1} \cup A_{2}$ is a maximal 2-partite set of $H$. The following lemma shows that some $\chi_{H}(V)$ covering of $V$ yields initial segment unions which are maximal $k$-partite sets for all $1 \leq k \leq \chi_{H}(V)$ whenever $|\mathfrak{F}|$ is finite.

Lemma 3 (Uniform set covering lemma). Let the set system $H=(V, \mathfrak{F})$ where $|\mathfrak{F}|$ is finite. Then there exists a minimum covering $A_{1}, A_{2}, \cdots$, $A_{\chi_{H}(V)}$ of $V, A_{i} \in \mathcal{F}$ for $1 \leq i \leq \chi_{H}(V)$, such that

(i) $\bigcup_{i=1}^{k} A_{i}$ is a maximal $k$-partite set for $1 \leq k \leq \chi_{H}(V)$,

(ii) $v \in \bigcup_{i=1}^{l(v)} A_{i}$ for all $v \in V$.

Proof. For $\chi_{H}(V)=1$, the result of the lemma is immediate. Assume condition (i) of the lemma to hold for all set systems $H=(V, \mathfrak{F})$ with finite $|\mathfrak{F}|$ and covering numbers $\chi_{H}(V) \leq n-1$ for a given $n \geq 2$, and let $H^{*}=$ $\left(V^{*}, \mathfrak{F}^{*}\right)$ have finite $\left|\mathfrak{F}^{*}\right|$ and covering number $\chi_{H^{*}}\left(V^{*}\right)=n$. Let $A_{1}^{*}, A_{2}^{*}, \cdots$, $A_{n}^{*}, A_{i}^{*} \in \mathcal{F}^{*}$, for $1 \leq i \leq n$, be a minimum covering of $V^{*}$. Let $B$ be a maximal $(n-1)$-partite set of $H^{*}$ such that $\bigcup_{i=1}^{n-1} A_{i}^{*} \subset B \subset V^{*}$. Form the set system $H_{B}=\left(B, \mathcal{F}_{B}\right)$, where $\mathcal{F}_{B}=\left\{S \mid S \neq \varnothing, S=S^{\prime} \cap B\right.$ for some $\left.S^{\prime} \in \mathcal{F}^{*}\right\}$. Then $\chi_{H_{B}}(B)=n-1$, and $\left|\mathcal{F}_{B}\right|$ is finite, so by our initial assumption there exists a covering $B_{1}, B_{2}, \cdots, B_{n-1}$ of $B$ with $B_{i} \in \mathcal{F}_{B}$ and $B_{i}=S_{i} \cap B$ for $S_{i} \in \mathcal{F}^{*}$ 
for $1 \leq i \leq n-1$ where $\bigcup_{i=1}^{k} B_{i}$ is a maximal $k$-partite set of $B$ for $1 \leq k \leq n-1$. Then $B=\bigcup_{i=1}^{n-1} B_{i} \subset \bigcup_{i=1}^{n-1} S_{i}$, and $\bigcup_{i=1}^{n-1} S_{i}$ is an $(n-1)$-partite set of $H^{*}$. However, $B$ was chosen to be a maximal $(n-1)$-partite set of $H^{*}$, so $B=\bigcup_{i=1}^{n-1} S_{i}$, and $B_{i}=S_{i}$ for all $i$. For $1 \leq k \leq n-1$, let $\bigcup_{i=1}^{k} B_{i} \subset D_{k} \subset V^{*}$ where $D_{k}$ is a maximal $k$-partite set of $H^{*}$. Now $D_{k} \cap B$ is a $k$-partite set of $H_{B}$, and $D_{k} \cap B=\bigcup_{i=1}^{k} B_{i}$, since $\bigcup_{i=1}^{k} B_{i}$ is a maximal $k$-partite set of $H_{B}$. Also $D_{k} \cap V-B=\varnothing$, since otherwise $D_{k} \cup \bigcup_{i=k+1}^{n-1} B_{i}$ would be an $(n-1)$-partite set of $H^{*}$ strictly containing $B$. Thus $\bigcup_{i=1}^{k} B_{i}$ is a maximal $k$-partite set of $H^{*}=\left(V^{*}, \mathcal{F}^{*}\right)$ for $1 \leq k \leq n-1$, hence $B_{1}, B_{2}, \cdots, B_{n-1}, A_{n}$ is a cover of $V^{*}$ satisfying condition (i) of the statement of the lemma. By induction, condition (i) then holds for all set systems $(V, \mathcal{F})$ with $|\mathfrak{F}|$ finite. Condition (ii) then follows from condition (i) and Corollary 2.1, proving the lemma.

Let $A_{1}, A_{2}, \cdots, A_{k}$ be a covering of $V$ for the bounded set system $H=$ $(V, \mathfrak{F})$. Any set $A \subset V$ must meet at least $\chi_{H}(A)$ members of this covering, and in particular if $C_{v}$ is a critical subset of $V$ containing $v$ with $l(v)=$ $\chi\left(C_{v}\right)$, then at least $l(v)$ members from $A_{1}, A_{2}, \cdots, A_{k}$ are needed to cover $C_{v}$. This motivates our definition that a covering $A_{1}, A_{2}, \cdots, A_{k}$ of $V$ for the set system $H=(V, \mathcal{F})$ is a uniform set covering for $H$ if $v \in \bigcup_{i=1}^{l(v)} A_{i}$ for all $v \in V$. Lemma 3 thus assures us that a uniform set covering always exists for any set system $H=(V, \mathcal{F})$ with finite $|\mathcal{F}|$. The bounded set system $H^{\prime}=\left(V^{\prime}, \mathcal{F}^{\prime}\right)$ described in equation (1) has $l(v)=1$ for all $v \in V^{\prime}$, yet $\chi_{H},\left(V^{\prime}\right)=2$. Thus $H^{\prime}$ has no uniform set covering, so all bounded set systems do not possess uniform set coverings.

A uniform set covering provides a minimum set covering for the whole of $V$. Conversely, it is evident for any finite set system $H$ that the methods employed in the inductive proof of Lemma 3 could be utilized recursively a long with any existing procedure for determining a minimum set covering of a finite set to yield a procedure for determining a uniform set covering of $H$. Thus the problem of determining a uniform set covering of a finite set system clearly has computational complexity which is equivalent, in the sense of existence or nonexistence of a polynomial bounded algorithm, to that of determining a minimum set covering of a finite set, and therefore belongs to a large class of finite but computationally difficult problems described in [3].

III. An application to graph theory. The un iform set covering lemma can be applied to the theory of graph colorings [5]. Given the graph $G=$ 
$(V, E)$, form the finite set system $H=\left(V, \mathcal{F}_{l}\right)$, where $V$ is the finite vertex set from $G$, and $\mathcal{F}_{I}$ is the set of independent sets of vertices of the graph $G$. For $A \subset V$, the covering number $\chi_{H}(A)$ thus corresponds to the chromatic number of the induced subgraph $\langle A\rangle$ of $G$. The critical set $C$ of $H=\left(V, \mathfrak{F}_{I}\right)$ thus corresponds to a color-critical subgraph $\langle C\rangle$ of $G$, and $l(v)$ becomes the largest chromatic number of any colorocritical subgraph of $G$ containing $v$. A Grundy function on a graph $G$ is an assignment of nonnegative integer values (colors) to the vertices of $G$ such that each vertex has an assigned value equal to the lowest nonnegative integer not occurring on any adjacent vertex of $G$. Let $\chi(G)$ denote the chromatic number of the graph $G$.

Corollary 3.1. There exists a Grundy function $g$ on the graph $G=(V, E)$ such that for every $v \in V$,

$$
g(v) \leq-1+\max _{A \subset V}\{\chi(\langle A\rangle) \mid v \in A,\langle A\rangle \text { is color-critical }\} .
$$

Proof. Applying Lemma 3 to the set system $\left(V, \mathscr{F}_{I}\right)$ of independent sets of the graph $G$, the independent sets $A_{1}, A_{2}, \cdots, A_{\chi(G)}$ may be determined so that $v \in \bigcup_{i=1}^{l(v)} A_{i}$ for all $v \in V$. Assigning members of $A_{i}$ the color value $i-1$ for $1 \leq i \leq \chi(G)$, a graph coloring is determined where each vertex receives a color value $\leq-1+l(v)=-1+\max _{A \subset V}\{\chi(\langle A\rangle) \mid v \in A,\langle A\rangle$ is colorcritical\}. If this color function on the vertices is not a Grundy function, color values on vertices may be lowered eventually yielding a Grundy function $g$ of $G$ which satisfies (4).

In [4] we derived the following result: For any graph $G=(V, E)$, there exists a Grundy function $g$ on $V$ such that for every vertex $v \in V$,

$$
g(v) \leq \max \left\{\lambda\left(G^{\prime}\right) \mid G^{\prime} \text { a subgraph of } G \text { containing } v\right\},
$$

where $\lambda\left(G^{\prime}\right)$ is the edge-connectivity of $G^{\prime}$. From a result of Dirac [2], Ore $[5$, p. 165] has shown that every color-critical graph $G$ satisfies $\chi(G) \leq$ $\lambda(G)+1$; thus our result from [4] is contained in Corollary 3.1.

\section{REFERENCES}

1. C. Berge, Graphes et hypergraphes, Dunod, Paris, 1970.

2. G. A. Dirac, The structure of $k-$ chromatic graphs, Fund. Math. 40 (1953), 42-55. MR 15, 640 .

3. R. M. Karp, Reducibility among combinatorial problems, Complexity of Computer Computations, R. E. Miller and J. W. Thatcher (editors), Plenum, New York, 1972, pp. 85-103.

4. D. W. Matula, Bounded color functions on graphs, Networks 2 (1972/73), 29-44. MR 45 \#6681. 
5. O. Ore, The four color problem, Pure and Appl. Math., vol. 27, Academic Press, New York, 1967. MR 36 \#74.

DEPARTMENT OF APPLIED MATHEMATICS AND COMPUTER SCIENCE, WASHINGTON UNIVERSITY, ST. LOUIS, MISSOURI 63130

DEPARTMENT OF COMPUTER SCIENCES, UNIVERSITY OF TEXAS, AUSTIN, TEXAS 78712

Current address: Department of Computer Science and Operations Research, Southern Methodist University, Dallas, Texas 75275 